\section{POS0607 PROMIS ASSESSMENT OF RESPONSE TO TREATMENT WITH GOLIMUMAB IV OR INFLIXIMAB IN RHEUMATOID ARTHRITIS PATIENTS: RESULTS FROM THE PHASE-4 AWARE STUDY}

C. Bingham ${ }^{1}$, S. Kafka ${ }^{2}$, S. Black ${ }^{3}$, S. Xu ${ }^{4}$, W. Langholff ${ }^{5}$, J. Curtis ${ }^{6} .{ }^{1}$ Johns Hopkins University, Division of Rheumatology, Baltimore, United States of

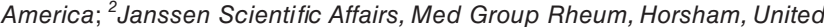
States of America; ${ }^{3} J a n s s e n ~ R \& D$ US, Immunology General, Horsham, United States of America: ${ }^{4}$ Janssen Research \& Development, Clinical Biostats, Spring House, United States of America; ${ }^{5}$ Janssen Research \& Development, Clinical Biostats, Horsham, United States of America; ${ }^{6}$ University of Alabama at Birmingham, Dept of Medicine, Birmingham, United States of America

Background: AWARE is a phase-4 observational study designed to provide real-world assessment of Golimumab (GLM) IV \& infliximab (IFX) in patients (pts) with rheumatoid arthritis (RA).

Objectives: To assess patient-reported aspects of social, mental, \& physical health through the $8^{\text {th }}$ infusion ( $\approx 1$ year of treatment) using Patient Reported Outcomes Measurement Information System (PROMIS), a validated, disease-agnostic set of health assessment instruments.

Methods: AWARE enrolled 1270 RA pts initiating treatment with GLM/IFX. The 52 week analysis set included pts with $\geq 1$-year treatment or those discontinued and, while enrolled, completed PROMIS-29 or PROMIS short form (SF) questionnaires. PROMIS instruments were administered at baseline \& prior to infusions $2,5, \& 8$. The raw score was converted into a standardized T-score with a mean of 50 and SD of 10.

Results: At baseline, treatment groups were balanced on demographics \& medical characteristics. Most pts were white $(87.0 \%$ GLM, $86.2 \%$ IFX)
\& female $(83.4 \%$ GLM, $82.4 \%$ IFX). Mean ages were $58.5 \pm 12.96$ years for GLM \& $59.6 \pm 13.24$ years for IFX. Overall, $35.3 \%$ GLM \& $42.9 \%$ IFX pts were bio-naïve. The proportion of GLM \& IFX pts with prior exposure to 1 or 2 biologics was similar; however, $20.1 \%$ GLM pts vs $10.8 \%$ IFX pts had exposure to $\geq 3$ biologics. Methotrexate use was similar between GLM $(76.4 \%)$ \& IFX pts $(75.0 \%)$. Based on mean PROMIS T-scores at baseline (Table 1), Fatigue, Pain Interference, \& Physical Function domains approached or exceeded 1 SD worse than those of general US population. Through the $8^{\text {th }}$ infusion, GLM- \& IFX-treated pts achieved meaningful improvement based on mean changes from baseline in most PROMIS-29 domains \& respective SFs with no significant difference between GLM and IFX. The percentage of GLM or IFX pts with improvements of $\geq 3$ $\geq 5$, or $\geq 10$ units change in T-scores increased from infusion 2 through infusion 8.

Conclusion: RA pts treated with GLM or IFX achieved comparable improvements across PROMIS-assessed social, mental, \& physical health. PROMIS-29 was able to detect change to subsequent anti-tumor necrosis factor- $\alpha$ therapies.

Disclosure of Interests: Clifton Bingham Consultant of: AbbVie, BMS, Eli Lilly, Gilead, Janssen, Pfizer, Regeneron/Sanofi, Grant/research support from: Bristol-Myers Squibb, Shelly Kafka Employee of: Janssen Research \& Development, LLC, Shawn Black Employee of: Janssen Research \& Development, LLC, Stephen Xu Employee of: Janssen Research \& Development, LLC, Wayne Langholff Employee of: Janssen Research \& Development, LLC, Jeffrey Curtis Consultant of: AbbVie, Amgen, Bristol-Myers Squibb, Corrona, Janssen, Lilly, Myriad, Pfizer, Regeneron, Roche, UCB, Grant/research support from: AbbVie, Amgen, Bristol-Myers Squibb, Corrona, Janssen, Lilly, Myriad, Pfizer, Regeneron, Roche, UCB

DOI: 10.1136/annrheumdis-2021-eular.1349

Table 1. Mean (SD) Change from Baseline PROMIS-29 Domain and Short Form T-Scores: 52 Week Analysis Set

\begin{tabular}{|c|c|c|c|}
\hline & GLM & IFX & LSM difference $(95 \% \mathrm{Cl})^{\star}$ \\
\hline Anxiety (4-item) & $\mathrm{N}=6$ & $\mathrm{~N}=570$ & \\
\hline Baseline & $53.4(10.13)$ & $54.6(10.53)$ & \\
\hline Change from baseline at infusion 8 & $\mathrm{~N}=223-2.6(8.10)$ & $\begin{array}{l}N=286 \\
-3.7(7.86)\end{array}$ & $-0.29(-1.54,0.97)$ \\
\hline $\begin{array}{l}\text { Depression (4-item) } \\
\text { Baseline }\end{array}$ & $\begin{array}{l}N=674 \\
51.9(9.83)\end{array}$ & $\begin{array}{l}N=574 \\
52.5(10.21)\end{array}$ & \\
\hline Change from baseline at infusion 8 & $\begin{array}{l}N=225 \\
-2.1(7.56)\end{array}$ & $\begin{array}{l}N=287 \\
-2.3(7.89)\end{array}$ & $0.49(-0.72,1.70)$ \\
\hline $\begin{array}{l}\text { Fatigue (4-item) } \\
\text { Baseline }\end{array}$ & $\begin{array}{l}N=671 \\
58.4(9.91)\end{array}$ & $\begin{array}{l}\mathrm{N}=574 \\
59.4(9.99)\end{array}$ & \\
\hline Change from baseline at infusion 8 & $\begin{array}{l}\mathrm{N}=225 \\
-3.4(8.72)\end{array}$ & $\begin{array}{l}N=281 \\
-3.1(7.77)\end{array}$ & $0.69(-0.64,2.03)$ \\
\hline $\begin{array}{l}\text { Short form Fatigue } 7 a \\
\text { Baseline }\end{array}$ & $\begin{array}{l}\mathrm{N}=681 \\
59.1(8.51)\end{array}$ & $\begin{array}{l}\mathrm{N}=576 \\
59.7(8.25)\end{array}$ & \\
\hline Change from baseline at infusion 8 & $\begin{array}{l}N=228 \\
-3.2(7.40)\end{array}$ & $\begin{array}{l}N=287 \\
-2.4(6.35)\end{array}$ & $1.01(-0.11,2.14)$ \\
\hline $\begin{array}{l}\text { Pain interference (4-item) } \\
\text { Baseline }\end{array}$ & $\begin{array}{l}N=679 \\
63.0(7.56)\end{array}$ & $\begin{array}{l}\mathrm{N}=574 \\
63.9(7.80)\end{array}$ & \\
\hline Change from baseline at infusion 8 & $\begin{array}{l}\mathrm{N}=227 \\
-4.2(8.23)\end{array}$ & $\begin{array}{l}\mathrm{N}=284 \\
-3.1(7.77)\end{array}$ & $1.84(0.55,3.13)$ \\
\hline $\begin{array}{l}\text { Short form Pain interference } 6 b \\
\text { Baseline }\end{array}$ & $\begin{array}{l}\mathrm{N}=680 \\
61.9(7.45)\end{array}$ & $\begin{array}{l}\mathrm{N}=576 \\
62.8(7.54)\end{array}$ & \\
\hline Change from baseline at infusion 8 & $\begin{array}{l}\mathrm{N}=228 \\
-3.8(7.88)\end{array}$ & $\begin{array}{l}N=287 \\
-3.2(6.67)\end{array}$ & $1.31(0.15,2.48)$ \\
\hline $\begin{array}{l}\text { Physical function (4-item) } \\
\text { Baseline }\end{array}$ & $\begin{array}{l}\mathrm{N}=678 \\
38.2(6.79)\end{array}$ & $\begin{array}{l}\mathrm{N}=571 \\
38.0(6.90)\end{array}$ & \\
\hline Change from baseline at infusion 8 & $\begin{array}{l}\mathrm{N}=224 \\
2.2(5.64)\end{array}$ & $\begin{array}{l}\mathrm{N}=283 \\
1.9(5.85)\end{array}$ & $-0.76(-1.73,0.21)$ \\
\hline $\begin{array}{l}\text { Sleep disturbance (4-item) } \\
\text { Baseline }\end{array}$ & $\begin{array}{l}\mathrm{N}=671 \\
54.6(8.72)\end{array}$ & $\begin{array}{l}\mathrm{N}=569 \\
\mathrm{~N}=55.5(8.61)\end{array}$ & \\
\hline Change from baseline at infusion 8 & $\begin{array}{l}\mathrm{N}=221 \\
-1.4(7.45)\end{array}$ & $\begin{array}{l}\mathrm{N}=281 \\
-1.7(7.61)\end{array}$ & $0.23(-0.96,1.42)$ \\
\hline $\begin{array}{l}\text { Social participation (4-item) } \\
\text { Baseline }\end{array}$ & $\begin{array}{l}\mathrm{N}=673 \\
43.7(8.40)\end{array}$ & $\begin{array}{l}\mathrm{N}=574 \\
42.9(8.77)\end{array}$ & \\
\hline Change from baseline at infusion 8 & $\begin{array}{l}\mathrm{N}=225 \\
3.2(8.15)\end{array}$ & $\begin{array}{l}\mathrm{N}=283 \\
3.4(7.48)\end{array}$ & $-0.10(-1.36,1.16)$ \\
\hline
\end{tabular}

*Least squares mean (LSM) difference \& confidence interval $(\mathrm{Cl})$ are based on analysis of covariance controlling for baseline PROMIS score using inverse probability of treatment weighted propensity score. 